\title{
The use of intensive radiological assessments in routine surveillance after treatment for head and neck cancer: an economic evaluation
}

\author{
Michela Meregaglia a, b*, John Cairns ${ }^{\text {a, c }}$, Lisa Licitra ${ }^{\text {d }}$, Paolo Bossi ${ }^{\text {d }}$
}

${ }^{a}$ Department of Health Services Research and Policy; Faculty of Public Health and Policy; London School of Hygiene and Tropical Medicine, 15-17 Tavistock Place, WC1H9SH, London (UK)

${ }^{\mathrm{b}}$ CeRGAS (Research Centre on Health and Social Care Management), Bocconi University, Via Roentgen 1, 20136, Milan (Italy)

${ }^{c}$ CCBIO (Center for Cancer Biomarkers), University of Bergen, Postboks 7804, N-5020 Bergen (Norway)

${ }^{\mathrm{d}}$ Head and Neck Medical Oncology Department, IRCCS Foundation National Cancer Institute, University of Milan, Via Venezian 1, 20133, Milan (Italy)

* Corresponding author: Michela Meregaglia, Via Roentgen 1, 20136 Milan (Italy). Tel. +39/02.5836.3677. Email: michela.meregaglia@unibocconi.it 


\section{ABSTRACT}

Background. There is uncertainty around the optimal surveillance of head and neck cancer patients following primary curative treatment. This study aims at assessing the cost-effectiveness of a post-treatment program of frequent radiological assessments (maximal approach) compared to a symptom-driven surveillance (minimal approach).

Materials and Methods. A decision-analytic Markov model is developed to assess the cost-utility of two alternative follow-up programs with a lifetime horizon. The two interventions differ in the number of radiological assessments (i.e. magnetic resonance imaging, computed tomography, and positron emission tomography) performed over a 5year period. Clinical and utility parameters are derived from published and unpublished literature and expert opinion. The cost analysis is conducted from the perspective of a major Italian region's healthcare system. Costeffectiveness results are expressed as incremental cost per life year gained (LYG) and per quality-adjusted life year (QALY) and checked against a cost-effectiveness threshold of €25,000-40,000 per QALY. One-way, twoway, and probabilistic sensitivity analyses are carried out.

Results: In the base-case analysis, an intensive program of radiological investigations leads to 0.10 additional QALYs (0.15 LYG) and an increase in costs of $€ 1,903$ per patient compared to a minimal option, resulting in an incremental cost of $€ 19,951 / \mathrm{Q} A L Y$ gained $(€ 13,123 / \mathrm{LYG})$. In probabilistic sensitivity analysis, $72 \%$ of the results lie below the $€ 40,000$ threshold $(55 \%$ below $€ 25,000)$.

Conclusions: An intensive post-treatment follow-up with scheduled radiological assessments over time might be cost-effective compared to symptom-driven surveillance in head and neck cancer patients. Further research is needed to check these results in empirical studies or real-world settings.

Keywords: head and neck cancer; follow-up; radiological assessments; Markov model; cost-utility analysis. 


\section{Introduction}

Head and neck cancer (HNC) is the sixth most common cancer worldwide; in Europe alone, around 143,000 people are diagnosed and more than 68,000 die each year because of the disease [1]. The incidence in Italy is about 16 cases per 100,000 [2]. Despite the routine introduction of combinedmodality treatment, the 5-year overall survival rate is $40 \%$ to $60 \%$ [2-4] and up to $50 \%$ of patients relapse with loco-regional or metastatic recurrences [4-6]; additionally, a constant rate of 2-3\% per year of second primaries is observed [7].

A few patients with loco-regional recurrences or second primaries can be salvaged by a potentially curative treatment (i.e. surgery or re-irradiation) [1, 4], while most are only suitable for palliative treatment usually including a combination of chemotherapeutics and anti-epidermal growth factor receptor drugs [8]. The prognosis for patients with recurrent or metastatic disease not eligible for curative treatment is very poor, with a median overall survival of around 10 months under the standard scheme of platinum-based chemotherapy plus cetuximab [9].

A post-treatment follow-up program is essential in the first few years after primary treatment to identify potentially curable relapses, as well as monitoring long-term therapy-related side effects. However, there is no consensus in the medical community around the optimal strategy. Published recommendations are mostly based on retrospective studies and expert opinions, whilst the added value of intensive radiological assessment over a scheme based on self-reported symptoms (e.g. pain, dysphagia, hoarseness) has not yet been confirmed in any prospective study.

This study evaluates the cost-effectiveness of an intensive follow-up strategy (maximal approach) versus a symptom-driven surveillance (minimal approach) using a modeling framework. 


\section{Materials and methods}

A decision-analytic Markov model is developed to assess the long-term health and economic consequences of two different surveillance schemes. A randomized controlled trial (HETeCo, clinicaltrials.gov identifier NCT02262221) is currently being conducted in Italy and Switzerland to compare an intensive versus a non-intensive follow-up program of equal length (i.e. 5 years). The trial started in 2014 and is expected to be completed by 2020; thus, it is mainly used to generate a research question, while most of the data are obtained from other sources.

\subsection{HETeCo trial}

The full trial protocol is available at clinicaltrials.gov. Briefly, patients with a diagnosis of clinical or pathological stage III-IV squamous HNC in the oral cavity, oropharynx, larynx, or hypopharynx and without evidence of disease six months after having received radiotherapy with curative intent (alone or with systemic therapy or in postoperative setting) are randomly allocated to one of two follow-up programs.

The non-intensive follow-up (arm A, minimal approach), designed according to National Comprehensive Cancer Network (NCCN) guidelines [10], comprises several outpatient visits during which patients receive both physical and fiber optic endoscopic examinations; laboratory tests are performed once a year. Radiological assessment through magnetic resonance imaging (MRI) or computed tomography (CT) is performed within six months of completion of treatment and then only at the occurrence of new signs or symptoms. Patients are contacted by phone between visits to monitor any health changes and instructed how to recognize them. 
The alternative strategy (arm B) is a more intensive follow-up (maximal approach) where outpatient visits and laboratory tests are performed similarly to arm A. Imaging tests are scheduled for all patients twice a year in the first two years and annually in the third and fourth years; MRI is preferred over CT for all sub-sites except for laryngeal cancer. Positron emission tomography (PET) scans are performed annually in the first three years in high-risk patients.

\subsection{Model structure}

The Markov state-transition model (Figure 1) simulates the experience of a hypothetical cohort of 1,000 patients after being treated for primary stage III-IV HNC; mean age and gender ratio are representative of the patients enrolled in the trial (until May 2016). All patients enter the model free of disease and move through the different health states according to a set of transition probabilities. Recurrent patients are divided based on the intent of the treatment received (i.e. potentially curative or palliative); patients treated with curative intent are assigned to either 'surgery' or 're-irradiation' states. Patients without progression remain in the 'no evidence of disease' states; the final, absorbing state is 'death'.

The cycle length of the model is one month with a lifetime horizon. Utility weights ranging from 0 (death) to 1 (perfect health) and costs are applied to the time spent in each health state. The model is run until the whole cohort (i.e. $>99 \%$ ) dies to estimate differences in life years gained (LYG), quality-adjusted life years (QALYs) and long-term costs associated with the two follow-up schemes under investigation in the HETeCo trial. 


\subsection{Clinical and utility parameters}

Transition probabilities between states are mainly derived from a combination of clinical parameters (Tables 1-2). The proportion of potentially salvageable recurrences in the study arm A (25\%) is derived from the literature [11], while the percentage in group B (50\%) is a clinical assumption of the study, which is intended to test whether a more intensive radiological assessment could detect a higher rate of salvageable relapses. The 'potentially curative treatment' state is assumed temporary, meaning that each patient can only remain in it for one cycle; surgery is considered the only potentially curative treatment for second primaries. The risk of relapsing after secondary treatment is estimated at $3 \%$ monthly, based on published studies [12-14]; this parameter is also consistent with the 0.009 weekly (i.e. 0.034 monthly) adopted by a previous cost-effectiveness model in Italy [15]. Any recurrence (or second primary) beyond the first is assumed to be treated with palliative intent only. Patients receiving palliative chemotherapy are assumed to have a median survival of 10 months, corresponding to a 1-year overall survival of around $43 \%$ (i.e. $6.6 \%$ monthly mortality) $[9,16]$. In each health state patients also experience a general risk of dying for reasons other than HNC; mortality rates for 5-year age groups are obtained from official statistics [17]. Annual probability values reported in the literature are transformed into monthly probabilities using the formula: $\mathrm{p}=1-\exp (-\mathrm{r} * \mathrm{t})$ where $p$ is probability, $r$ is rate and $t$ is the time expressed in months [18].

The utility parameters are summarized in Table 3. An average utility value for the "no evidence of disease' state (i.e. at recruitment) is calculated from the EuroQol 5 Dimensions 5 Levels (EQ-5D-5L) trial data using the English tariff set. The same value (i.e. 0.85) is confirmed by a cross-sectional study recruiting a comparable population [19]. Utility values for all other states are identified by a systematic literature review [20]. 


\subsection{Cost data}

The cost analysis is conducted from the perspective of a major Italian region (i.e. Lombardy) healthcare system. Unit costs for hospital admissions, specialist visits, radiological exams, laboratory tests and outpatient treatment regimens are from diagnosis-related groups (DRGs) and other regional tariffs (year 2016) (Table 4). The cost of each follow-up program (A or B) performed in the 'no evidence of disease' state is calculated for 5 years (i.e. standard length of follow-up) and reported in Table 5, according to the description provided in the trial protocol. Patients surviving the 'potentially curative treatment' states are assumed to be monitored within a program resembling the less intensive scheme (arm A). Re-irradiation is assumed to involve a cycle of intensity modulated radiation therapy (IMRT) sessions based on current practice. Standard platinum-based chemotherapy supplemented with 5-fluoraouracil (5FU) and cetuximab until one month before death is assumed for the 'palliatively treated recurrence' state; additionally, an average cost of dying for HNC is assigned to each patient entering this state based on the estimated consumption of formal end-of-life care (i.e. home-based assistance and hospital care). A synthesis of monthly cost values for all health states is reported in Table 6.

\subsection{Cost-effectiveness analysis}

Health outcomes (i.e. LYG, QALYs gained) and total costs are combined into an incremental cost-

effectiveness ratio $\left(\mathrm{ICER}=\frac{\operatorname{Cost}_{B}-\operatorname{Cost}_{A}}{\mathrm{LYs}_{\mathrm{B}}-\mathrm{LYs}_{\mathrm{A}}}\right)$ and cost-utility ratio $\left(\mathrm{ICUR}=\frac{\operatorname{Cost}_{B}-\operatorname{Cost}_{A}}{\mathrm{QALYs}_{\mathrm{B}}-\mathrm{QALYs}_{\mathrm{A}}}\right)$ to represent the incremental cost of achieving one unit of health outcome when an intensive follow-up strategy (arm B) replaces a less intensive one (arm A). The ICUR obtained is compared with the range of $€ 25,000$ $€ 40,000$ recommended by the Italian Health Economics Association [21]. All costs and outcomes are discounted at 3\% (converted to 0.247\% monthly) and expressed in Euro (€) 2016. 


\subsection{Sensitivity analyses}

One-way sensitivity analyses explore the robustness of the base-case results by varying some key model parameters one at a time. For example, the risk of overall relapse during follow-up is varied between

$20 \%$ and $50 \%$. The impact of different risks of dying because of toxicities following re-irradiation is explored using values from a systematic review [8]. We also examine the alternative that patients surviving the 'potentially salvageable recurrence' states have the same follow-up intervention (A or B) received in the 'no evidence of disease' state after primary treatment.

Additionally, a two-way sensitivity analysis is performed to assess the simultaneous effect of varying the proportion of salvageable recurrences in the two arms, with all else unchanged in the model. This analysis is performed because of the uncertainty surrounding this parameter based on clinical opinion and not yet confirmed within the ongoing trial.

Finally, a probabilistic sensitivity analysis is performed using Monte Carlo simulation with 5,000 random iterations from the distributions assigned to the model parameters. A beta distribution is chosen for probabilities and utilities, and a gamma distribution for costs (except for official tariffs, which are fixed in the model). A cost-effectiveness scatterplot illustrates the uncertainty surrounding the base-case ICUR, while cost-effectiveness acceptability curves (CEAC) quantify the probability of the intensive follow-up being cost-effective at different thresholds.

The decision model is implemented in Microsoft Excel 2013 with the support of @ RISK software (Palisade Corp) for the sensitivity analyses. 


\section{Results}

The baseline cost-effectiveness results yield an ICUR of $€ 19,951$ per QALY gained and an ICER of $€ 13,123$ per LYG (Table 7). In univariate sensitivity analysis (Table 8 ), the recurrence risk over the 5year follow-up is inversely proportional to the ICUR. Other variables including re-treatment-related mortality, cost of head and neck surgery and discounting have a limited effect on the results. In Figure 2, the cost-effectiveness of the intensive follow-up (arm B) increases with the positive difference between the "curability" of arm B and arm A, reaching a maximum value of $€ 6,330 / \mathrm{QALY}(€ 4,163 / \mathrm{LYG})$ when this parameter is equal to 0.7 in arm $\mathrm{B}$ and 0.1 in arm $\mathrm{A}$ and a minimum value of $€ 113,354 / \mathrm{Q} A L Y$ $(€ 74,561 / \mathrm{LYG})$ when the difference between the two "curability" rates is only 0.05 .

In the cost-effectiveness plane (Figure 3), most ICURs (72\%) are to the right of the $€ 40,000$ willingnessto-pay for Italy; even considering the lowest threshold (i.e. €25,000), the maximal approach is costeffective in more than $50 \%$ of simulations. None of the simulations fell in the left side of the graph implying a negative difference in QALYs. In Figure 4, the CEAC shows that at a willingness-to-pay equal to zero almost $5 \%$ of the simulations report a cost-saving result.

\section{Discussion}

There is no agreement on a common follow-up strategy in HNC across clinical guidelines worldwide. Among them, the NCCN guidelines do not recommend routine imaging in the absence of symptoms [10], while clinical practice in Italy is heterogeneous and sometimes involves more intensive programs. The addition of routine MRI, CT and PET scans to the scheduled clinical examinations might increase the detection accuracy of recurrent HNC in patients, especially the asymptomatic ones. However, the 
effectiveness (and cost-effectiveness) of more intensive follow-up schemes has never been shown with rigorous methods.

The current model predicts costs and outcomes in terms of QALYs and survival gains of two alternative follow-up programs in $\mathrm{HNC}$, corresponding to the arms of an ongoing clinical study. In this model, more intensive follow-up (maximal approach) appears cost-effective with a cost per QALY gained of $€ 19,951$, and more than two-thirds of the Monte Carlo simulations falling below the willingness-to-pay of $€ 40,000$. Moreover, the two-way sensitivity analysis shows that a difference in the proportion of potentially salvageable recurrence of about 0.15 between the two programs is sufficient to obtain an acceptable ICUR for arm B. The intermediate findings are comparable with data reported in the literature. For example, the 5-year survival is equal to $58 \%$ and $60.5 \%$ in arm $\mathrm{A}$ and arm $\mathrm{B}$, respectively, which is consistent with the epidemiological data [2-4]. Moreover, the average stay of 13-14 months in the palliative treatment state is aligned with the median overall survival (i.e. 10 months) reported by Vermorken [9], since survival times are positively skewed.

A recent study [22] systematically reviewed economic evaluations comparing alternative follow-up programs in cancer. Since no study considered HNC, and the incremental costs per QALY or LYG were rarely reported, comparisons with our results are not straightforward. However, we calculated an incremental cost per salvageable recurrence of $€ 20,249$, which coincides with the value reported (i.e. $£ 18,077$ ) by a modeling study comparing intensive versus standard surveillance for colorectal cancer in UK [23].

As a modeling study, the analysis inevitably represents a simplification of the real world. First, we assume that cancer-related deaths occur only during the active disease and patients without any cancer relapse experience the same risk of dying as the general population at the same age. However, previous 
studies have reported an extra-mortality risk for HNC survivors [24], that may lead to lower ICER/ICUR if this information is incorporated into the model. Second, we do not consider second primary tumors not in the head and neck region and reported by previous studies [25-27] in the lung, esophagus, and colon. Third, the possibility of combined salvage treatment (e.g. surgery followed by re-irradiation) [28] and the use of radiotherapy with palliative intent [29] are disregarded, as well as any re-treatment failures other than death, such as non-fatal toxicities [8] or residual disease after salvage surgery. Fourth, this study does not account for patient's anxiety and discomfort, nor for potential toxicities related to the use of PET and CT, and the risk of false-positive imaging leading to further costly investigations or unnecessary treatments $[25,30]$. Fifth, the costs of follow-up are spread out over a 5-year period, thus the monthly cost represents an average value; however, we derive two different estimates for follow-up $\mathrm{B}$, since most radiological assessments are performed during the first three years. Finally, the analysis is limited to direct healthcare costs, thus ignoring patient's out-of-pocket costs and productivity losses due to frequent travels to the hospital; moreover, the cost of informal care may be substantial during the terminal disease stages, when $91 \%$ of patients are estimated to be cared for at home [31].

This study is the first to investigate strategies of different intensity for monitoring patients after completion of treatments for primary HNC from a health economics perspective. Although a definite answer awaits the completion of the trial, the model shows that an intensive surveillance scheme may well be cost-effective in Italy. The trial protocol is currently under review, with a low-dose chest CT being included annually in heavy smokers according to the lung cancer screening NCCN guidelines [32]. Different results might be obtained by using alternative cost data, thus similar evaluations in other countries are valuable. Further research evaluating the benefits of a risk-adapted follow-up according to demographic, clinical or biomolecular factors is also warranted. 
Conflict of interest statement: None declared.

Funding source: This research did not receive any specific grant from funding agencies in the public, commercial, or not-for-profit sectors. No writing assistance has been used in drafting this article.

Acknowledgments: The main clinical trial (HETeCo, clinicaltrials.gov identifier NCT02262221) inspiring this evaluation has been possible thanks to the Swiss Bridge Award 2013. The authors also thank Prof Alessia Melegaro and Dr Carlo Federici (Bocconi University) who provided methodological advice for this study. 


\section{REFERENCES}

[1] Vermorken JB, Specenier P. Optimal treatment for recurrent/metastatic head and neck cancer. Ann Oncol 2010; 21 Suppl 7: vii252-vii261.

[2] AIOM, AIRTUM. I numeri del cancro in Italia 2015. Available at: http://www.registritumori.it/PDF/AIOM2015/I_numeri_del_cancro_2015.pdf

[3] Braakhuis BJ, Leemans CR, Visser O. Incidence and survival trends of head and neck squamous cell carcinoma in the Netherlands between 1989 and 2011. Oral Oncol 2014;50(7):670-5.

[4] Schenker Y, Arnold RM, Bauman JE, et al. An enhanced role for palliative care in the multidisciplinary approach to high-risk head and neck cancer. Cancer 2016;122(3):340-3.

[5] de Andrade DA, Machiels JP. Treatment options for patients with recurrent or metastatic squamous cell carcinoma of the head and neck, who progress after platinum-based chemotherapy. Curr Opin Oncol 2012; 24(3):211-7.

[6] Harrington K, Temam S, Mehanna H, et al. Postoperative adjuvant lapatinib and concurrent chemo-radiotherapy followed by maintenance lapatinib monotherapy in high-risk patients with resected squamous cell carcinoma of the head and neck: a phase III, randomized, double-blind, placebo-controlled study. J Clin Oncol 2015;33(35):4202-9.

[7] Choe KS, Haraf DJ, Solanki A, et al. Prior chemo radiotherapy adversely impacts outcomes of recurrent and second primary head and neck cancer treated with concurrent chemotherapy and re-irradiation. Cancer 2011; 117(20):4671-8. 
[8] Strojan P, Corry J, Eisbruch A, et al. Recurrent and second primary squamous cell carcinoma of the head and neck: when and how to re-irradiate. Head Neck 2015;37(1):134-50.

[9] Vermorken JB, Mesia R, Rivera F, et al. Platinum-based chemotherapy plus cetuximab in head and neck cancer. N Engl J Med 2008; 359(11):1116-27.

[10] Adelstein D, Gillison ML, Pfister DG, et al. NCCN Guidelines Insights: Head and Neck Cancers, Version 2.2017. J Natl Compr Canc Netw 2017; 15(6):761-770.

[11] Temam S, Pape E, Janot F, et al. Salvage surgery after failure of very accelerated radiotherapy in advanced head-and-neck squamous cell carcinoma. Int J Radiat Oncol Biol Phys 2005; 62(4):1078-83.

[12] Zafereo ME, Hanasono MM, Rosenthal DI, et al. The role of salvage surgery in patients with recurrent squamous cell carcinoma of the oropharynx. Cancer 2009; 115(24):5723-33.

[13] Biagioli MC, Harvey M, Roman E, et al. Intensity-modulated radiotherapy with concurrent chemotherapy for previously irradiated, recurrent head and neck cancer. Int J Radiat Oncol Biol Phys 2007; 69(4): 1067-73.

[14] Sher DJ, Haddad RI, Norris Jr CM, et al. Efficacy and toxicity of reirradiation using intensitymodulated radiotherapy for recurrent or second primary head and neck cancer. Cancer 2010; 116(20):4761-8

[15] Liberato NL, Rognoni C, Rubrichi S, et al. Adding docetaxel to cisplatin and fluorouracil in patients with unresectable head and neck cancer: a cost-utility analysis. Ann Oncol 2012; 23(7): 1825-32. 
[16] Won YW, Park YH, Ahn MJ, et al. A phase II study of combination chemotherapy with capecitabine and cisplatin in patients with metastatic or recurrent squamous cell carcinoma of the head and neck. Ann Oncol 2011; 22(2):417-23.

[17] Istituto Nazionale di Statistica (ISTAT). Tavole di mortalità per classi di età (2015). Available at: http://dati.istat.it/Index.aspx?DataSetCode=DCIS_MORTALITA1 (accessed February 2017)

[18] Briggs A, Sculpher M, Claxton K. Decision Modelling for Health Economic Evaluation (Handbooks in Health Economic Evaluation), 1st edition. Oxford University Press 2006.

[19] Ramaekers BL, Joore MA, Grutters JP, et al. The impact of late treatment-toxicity on generic health-related quality of life in head and neck cancer patients after radiotherapy. Oral Oncol 2011; 47(8):768-74.

[20] Meregaglia M, Cairns J. A systematic literature review of health state utility values in head and neck cancer. Health Qual Life Outcomes 2017 Sep 2;15(1):174.

[21] Fattore G. Proposta di linee guida per la valutazione economica degli interventi sanitari in Italia. Pharmacoeconomics Ital Res Articles 2009; 11: 83.

[22] Meregaglia M, Cairns J. Economic evaluations of follow-up strategies for cancer survivors: a systematic review and quality appraisal of the literature. Expert Rev Pharmacoecon Outcomes Res 2015;15(6):913-29.

[23] MacAfee DAL, Whynes DK, Scholefield JH. Risk-stratified intensive follow up for treated colorectal cancer - realistic and cost saving? Colorectal Dis 2007; 10:222-30. 
[24] Massa ST, Osazuwa-Peters N, Christopher KM, et al. Competing causes of death in the head and neck cancer population. Oral Oncol 2017; 65: 8-15.

[25] Imbimbo M, Bergamini C, Botta L, et al. Surveillance of head and neck cancer patients: clinical and radiological exams or symptom-driven follow-up? Radiother Oncol 2013; 106(Suppl 1): S16.

[26] González-Botas H, Vázquez V, Barro V. Second primary tumors in head and neck cancer. Acta Otorrinolaringol Esp 2016;67(3):123-9.

[27] Priante AV, Castilho EC, Kowalski LP. Second primary tumors in patients with head and neck cancer. Curr Oncol Rep 2011;13(2):132-7.

[28] Ho AS, Zumsteg ZS, Meyer A, et al. Impact of flap reconstruction on radiotoxicity after salvage surgery and re-irradiation for recurrent head and neck cancer. Ann Surg Oncol 2016; 23 (Suppl 5):850-857.

[29] Paleri V, Kelly GC. Re-irradiation with concurrent chemotherapy in recurrent head and neck cancer: a decision analysis model based on a systematic review. Clin Otolaryngol 2008; 33(4):331-7.

[30] Huntington SF, Svoboda J, Doshi JA. Cost-effectiveness analysis of routine surveillance imaging of patients with diffuse large B-cell lymphoma in first remission. J Clin Oncol 2015; 33(13): 1467-74. 
[31] Beccaro M, Costantini M, Merlo DF; ISDOC Study Group. Inequity in the provision of and access to palliative care for cancer patients. Results from the Italian survey of the dying of cancer (ISDOC). BMC Public Health 2007; 7:66.

[32] Wood DE, Kazerooni E, Baum SL, et al. Lung cancer screening, version 1.2015: featured updates to the NCCN guidelines. J Natl Compr Canc Netw 2015; 13(1):23-34.

[33] Louis DZ, Hegarty SE, Leoni M, et al. Variation in hospital utilization at the end of life for patients with cancer in the Emilia-Romagna region of Italy. Tumori 2016; 102(6): 614-620.

[34] Penel N, Amela EY, Mallet Y, et al. A simple predictive model for postoperative mortality after head and neck cancer surgery with opening mucosa. Oral Oncol 2007; 43(2): 174-80.

[35] Szabo SM, Dobson RL, Donato BMK, et al. The quality-of-life impact of head and neck cancer: preference values from the Canadian general public. Health Outcomes Res Med 2012; 3(1): e11-e23.

[36] Noel CW, Lee DJ, Kong Q, et al. Comparison of health state utility measures in patients with head and neck cancer. JAMA Otolaryngol Head Neck Surg 2015;141(8):696-703.

[37] Pannuti R. Qualità di vita del malato terminale e sostenibilità delle cure palliative: l'integrazione tra sanità pubblica e privato sociale. Evidence 2015; 7(9): e1000118.

[38] Francisci S, Guzzinati S, Mezzetti M, et al. Cost profiles of colorectal cancer patients in Italy based on individual patterns of care. BMC Cancer 2013; 13: 329.

[39] Patel PR, Salama JK. Reirradiation for recurrent head and neck cancer. Expert Rev Anticancer Ther 2012; 12(9): 1177-89. 
[40] Neumann PJ, Ganiats TG, Sanders GD, Russell LB, Siegel JE. Cost-Effectiveness in Health and Medicine. Second Edition. Oxford University Press 2017. 